\title{
Influence of clinical usage of GT and GTX files on cyclic fatigue resistance
}

\author{
A. Arias, J. J. Perez-Higueras \& J. C. de la Macorra \\ Department of Conservative Dentistry, School of Dentistry, Complutense University of Madrid, Madrid, Spain
}

\begin{abstract}
Arias A, Perez-Higueras JJ, de la Macorra JC. Influence of clinical usage of GT and GTX files on cyclic fatigue resistance. International Endodontic Journal.
\end{abstract}

\begin{abstract}
Aim To compare static cyclic fatigue resistance of unused, unused and sterilized and clinically used conventional NiTi GT and M-Wire GTX files.

Methodology One hundred and sixty new files (80 GT and 80 GT series X) were divided into four control groups and four experimental groups $(n=20$ each). Control groups were new unused files and new sterilized files. In the experimental groups, instruments were used in three (GT1 and GTX1 groups) or four molars (GT2 and GTX2 groups). Cyclic fatigue resistance was tested in stainless steel curved canals $\left(60^{\circ}\right.$, $r=3 \mathrm{~mm})$. Each file was rotated until fracture (300 rpm, $2 \mathrm{~N} \mathrm{~cm}$ torque). Time-to-fracture (s) was registered. Mean life, eta and beta parameters of their Weibull distributions were calculated.
\end{abstract}

Results Unused and sterilized GTX files will last significantly longer than GT files with a probability of
$75 \%$ and $65 \%$, respectively; whilst mean life was significantly longer for GT than for GTX in used files with a probability of $68 \%$. Sterilized GT files will last longer than unused files with a probability of $66 \%$. In both brands, unused and sterilized files will last significantly longer than files used clinically with a probability higher than 98\%. The probability that GT will last longer after being used in three rather than four molars was $62 \%$ (statistically significant) and 52\% (not statistically significant) for GTX.

Conclusions GTX files had an extended cyclic fatigue life when compared with GT when they were unused or unused and sterilized, but GT were significantly more resistant to cyclic fatigue after clinical usage than GT series X files $3 \mathrm{~mm}$ from the tip. Sterilization of files enhanced the cyclic fatigue resistance of GT instruments. Clinical use of files diminished cyclic fatigue resistance.

Keywords: cyclic fatigue, GT, GTX, M-Wire, NiTi rotary files.

Received 24 October 2012; accepted 19 May 2013

\section{Introduction}

Due to their elasticity and cutting efficiency (Walia et al. 1988) and their effectiveness in preparing canals (Glosson et al. 1995, Short et al. 1997), nickeltitanium (NiTi) rotary files have become a popular when shaping root canals (Bird et al. 2009). Despite these advantages, fracture of NiTi rotary files caused by cyclic fatigue has remained a primary concern in

Correspondence: José C. de la Macorra, Department of Conservative Dentistry, School of Dentistry, Complutense University, Plaza Ramón y Cajal s/n, Ciudad Universitaria, 28040 Madrid, Spain (e-mail: macorra@ucm.es). endodontics practice because they tend to break unexpectedly as a result of the alternating tension-compression cycles to which they are subjected, usually when rotated near the apical third of a root canal with a large curvature (Sattapan et al. 2000).

Manufacturers have changed cross-sectional designs and geometrical traits of NiTi instruments to improve cyclic fatigue resistance. Another strategy to achieve this goal is the use of new alloys: new manufacturing processes optimize the microstructure of NiTi through a novel thermomechanical processing (Ye \& Gao 2012), producing a new alloy, M-Wire (Sportswire LLC, Langley, OK, USA), with a substantial increase in flexibility (Johnson et al. 2008). Endodontic instruments manufactured with M-Wire 
are expected to have higher strength and wear resistance than similar instruments made of conventional superelastic NiTi wires because of its unique nanocrystalline martensitic microstructure (Ye \& Gao 2012).

GT and GT series X files (both manufactured by Dentsply, Tulsa Dental Specialities, Tulsa, OK, USA) are two instruments that have the same U-shaped cross-section, passive rounded tip geometry and limited maximum flute diameter. However, GT series X was designed to improve the characteristics of GT files through two significant changes: the land widths vary along the length of the file, and it is made of the new M-Wire alloy (Buchanan 2008).

Apart from many other factors including the radius, the degree of the root canal curvature or the design of the instrument, that affect cyclic fatigue resistance, clinical use of rotary files have been shown to decrease this resistance (Gambarini 2001, Fife et al. 2004, Bahia \& Buono 2005, Plotino et al. 2006, Ounsi et al. 2007, Aydin et al. 2010). Not only the resistance to $\mathrm{CF}$ but also the final preparation of the canal is affected when files are reused (Ounsi et al. 2011). However, due to the relatively high cost of NiTi rotary files and against manufacturers' recommendations, clinicians frequently reuse files, despite the enhanced the risk of metal fatigue (Bird et al. 2009).

How clinical use affects torsional resistance of GT and GTX files has been studied (Kell et al. 2009). However, no studies have compared the cyclic fatigue resistance between GT files and GT series X after clinical use.

It would be interesting to assess how cyclic fatigue resistance of M-Wire alloy files is affected by clinical usage, compared with traditional NiTi, considering that unused M-Wire GTX files have shown higher cyclic fatigue resistance than unused NiTi GT files (Larsen et al. 2009, da Cunha Peixoto et al. 2010, Solaiman et al. 2010). If resistance of reused GTX files were higher, it would allow more canals to be shaped with a lower risk of fracture compared with traditional NiTi GT files. Therefore, the aim of this study is to compare cyclic fatigue resistance of conventional NiTi GT and M-Wire GT series X files after clinical usage.

\section{Materials and methods}

This research was conducted with the approval of the Ethics Committee of Clinical Research of Saint Carlos Hospital, Madrid.

In total, 280 consecutive patients who needed primary root canal treatment in a molar with roots having a moderate angle of curvature (10-20 $)$ were enrolled. All patients were informed of the aims and design of the study, and written consent was obtained before their enrolment. All the root canal treatments were performed by the same endodontist (AA).

A sample of 80 size 30, 0.06 taper new files (40 GT and 40 GT series X) were sterilized and divided in four groups: GT1 (20 GT), GT2 (20 GT), GTX1 (20 GTX) and GTX2 (20 GTX). GT1 and GTX1 were used to shape the canals of three molars and GT2 and GTX2 in four molars. The number of canals treated with each individual file was also registered. After being used, their resistance to cyclic fatigue was tested. All the files had a tip diameter of $0.30 \mathrm{~mm}$ and a $6 \%$ continuous taper.

Shaping protocol was as follows: manual shaping to size 20 with K-Flexofile files, ProTaper S1, ProTaper S2, size 20, 0.06 taper GT/GTX and size 30, 0.06 taper GT/GTX (all files from Dentsply Maillefer, Ballaigues, Switzerland). All rotary files reached working length previously determined by a Root ZX electronic apex locator (J Morita Europe GmbH, Frankfurt, Germany) and confirmed radiographically. During the whole shaping procedure, $\mathrm{NaOCl} 5.25 \%$ was used as the irrigant.

After each treatment, files were cleaned using a gauze soaked in $5.25 \% \mathrm{NaOCl}$, packaged in a sterilization envelope with indicators that change colour to ensure the optimal functioning of the autoclave, and the number of treated molars and canals was annotated in the envelope.

Instructions of the autoclave Europa B xp (Tecno Gaz S.p.A., Parma, Italy) were followed for the sterilization process. The programme ' $134{ }^{\circ} \mathrm{C}$ packaged' cycle for instruments was chosen: the time exposed was $5 \mathrm{~min}$ and the drying time $12 \mathrm{~min}$. Warmingup time and vacuum fractionation could vary between 25 and $30 \mathrm{~min}$. Quality of the sterilization was monitored by physical, chemical and biological methods.

Another sample of 80 size 30, 0.06 taper new files (40 GT and 40 GT series X) was used as controls. They were divided in four groups. In two groups, new unused files were tested: GT-C1 (20 GT) and GTX-C1 (20 GTX). In the other two groups, new unused files were wiped with $\mathrm{NaOCl} 5.25 \%$ and sterilized three times with the same protocol as above: GT-C2 (20 GT) and GTX-C2 (20 GTX).

Cyclic fatigue resistance of all was tested at $3 \mathrm{~mm}$ from the tip. Diameter at the tested length was $0.48 \mathrm{~mm}$. 


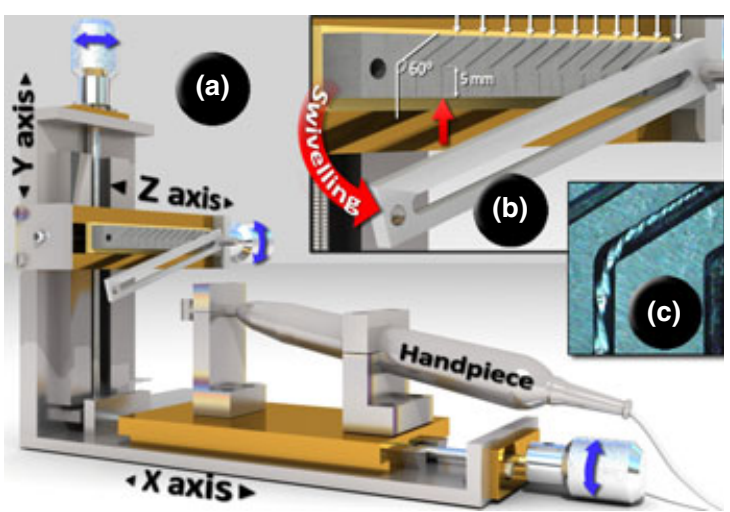

Figure 1 Cyclic fatigue testing device. Insert a: general view. Three axes are shown. Insert b: swivelling motion of grooved stainless steel top face cover and carved open semi-canals (white arrows). Red arrow points to the entrance of a canal. Insert c: image of the bending point of a file before testing.

\section{Cyclic fatigue test device}

Cyclic fatigue was tested in a device previously used and described (Arias et al. 2012). It consisted of a hardened stainless steel piece with 11 carved open semi-canals (white arrows, insert B in Fig. 1) with a depth $0.1 \mathrm{~mm}$ greater than their width and diameters ranging from 0.4 to $1.4 \mathrm{~mm}$. Every semi-canal had two straight portions, joined by a $60^{\circ}$ curvature (3-mm radius, insert B, Fig. 1). The first 5-mm straight vertical portion of the canal was the entrance (red arrow in insert B, Fig. 1), whilst in the second 4$\mathrm{mm}$ straight portion rested the tip of the file (insert C, Fig. 1).

The canal selected to test cyclic fatigue was the immediately wider (to the nearest $0.1 \mathrm{~mm}$ ) to the diameter of the file at the entrance of the semicanal. The diameter of the files at this point was 0.78 , so the canal of $0.8 \mathrm{~mm}$ width was used (insert B, Fig. 1).

The hardened stainless steel part was attached to a base that allowed the position of files in the three axes of space be adjusted (insert A in Fig. 1). The $x$-axis platform held the dental handpiece, sliding back and forth on a twisted spindle manoeuvred by a knob, approaching or separating from a second platform that held the stainless steel carved portion. This second platform had two knobs, each one allowing the adjustment of the vertical ( $y$ axis) or the depth ( $z$ axis) positions of the canals.

A swivelling, grooved stainless steel top face cover allowed for visualization of the files whilst rotating in the canal and served to protect the operator (insert B, Fig. 1).

\section{Positioning and testing of files}

Files were placed in the handpiece (insert A, Fig. 1) and inserted into the canal; the top face cover was swung and secured (insert B, Fig. 1). Both the canal and the file were lubricated with synthetic oil (Singer All-purpose oil; Singer Sewing Company, Barcelona, Spain) to minimize friction.

The files were rotated at $300 \mathrm{rpm}$ and with a torque of $2 \mathrm{~N} \mathrm{~cm}$ in an X-Smart device (Dentsply Maillefer, Ballaigues, Switzerland).

The motor and a $1 / 100 \mathrm{~s}$ chronometer were activated simultaneously. The file was monitored through the face cover during the test until fracture. The chronometer was then stopped, and the time (s)to-fracture was registered.

\section{Statistical analysis}

A Weibull analysis (Weibull ++ 7; Reliasoft Corporation, Tucson, AR, USA) was used to calculate different parameters (and their 95\%) CI for each group:

- Mean life (s). The expected or average time-tofailure.

- Beta, slope or shape parameter (dimensionless). The value is equal to the slope of the regressed line in the Weibull data plot. Different slopes imply different classes of failure modes, and are related to the quality of the designing of the material.

- Eta (s), characteristic life or scale parameter. The expected time that $63.2 \%$ of the files will reach without breakage, that is, the probability of failure being 0.63 at this time.

\section{Results}

The distribution of reliability versus time per group is shown in Fig. 2.

The mean (SD) number of shaped canals per group and the results for beta and eta (in seconds) parameters and mean life (in seconds) and 95\% CIs are shown in Table 1.

When mean life was compared between control and experimental groups, mean life was significantly higher in control groups for both GT and GTX. The probability that GT-C1 and GT-C2 will last longer than GT1 and GT2 was higher than $98 \%$, and the 


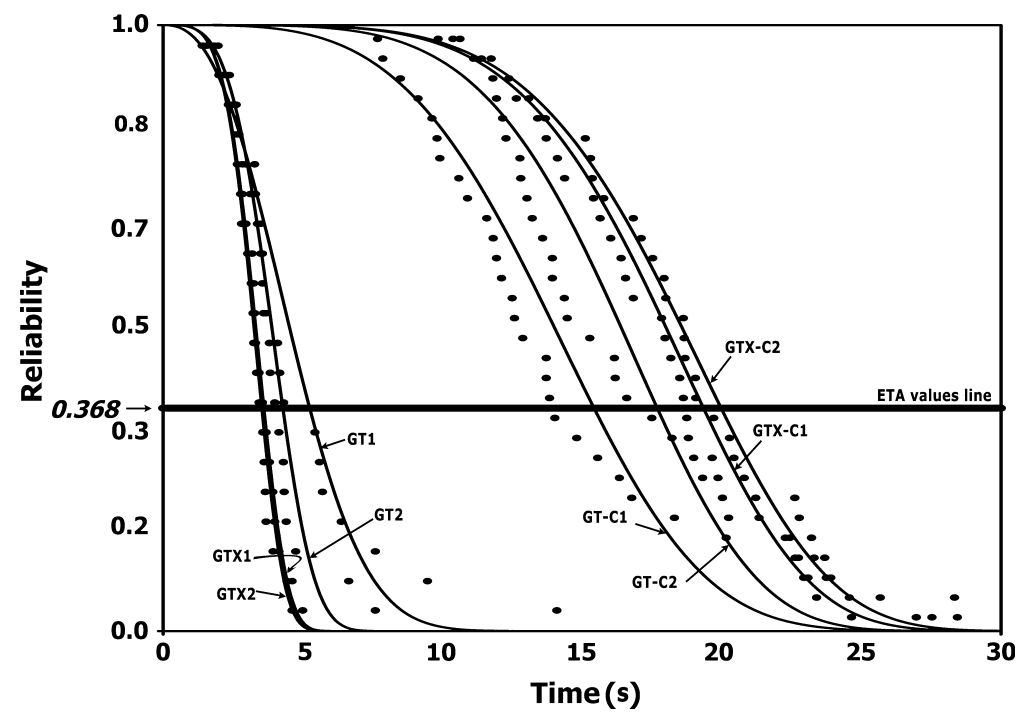

Figure 2 Weibull analysis reliability plot. The vertical scale is reliability, that is, the probability of survival. The horizontal scale is the time-to-failure (s). The horizontal thick dotted line represents Eta values, that is, the time (s) at which $63.2 \%$ of the files will survive.

Table $1 \mathrm{~N}$, mean (SD) number of shaped canals per group and results for beta and eta (in seconds) parameters and mean life (in seconds) and 95\% CIs

\begin{tabular}{llclcr}
\hline Group & $n$ & $\begin{array}{c}\text { \# of shaped canals } \\
\text { (mean (SD)) }\end{array}$ & Beta $(95 \% \mathrm{Cl})$ & Eta $(95 \% \mathrm{Cl})$ & Mean life $(95 \% \mathrm{Cl})$ \\
\hline GT-C1 & 20 & - & $3.8(3-4)$ & $15.4(13.8-17.2)$ & $14(12.3-15.9)$ \\
GT-C2 & 20 & - & $4.9(3.8-6.3)$ & $17.7(16.2-19.3)$ & $16.2(14.6-18)$ \\
GT1 & 20 & $11.5(0.8)$ & $2.5(1.9-3.3)$ & $5.3(4.3-6.5)$ & $4.7(3.7-5.9)$ \\
GT2 & 20 & $13.6(0.7)$ & $3.8(2.9-5.1)$ & $4.3(3.8-4.9)$ & $3.9(3.3-4.5)$ \\
GTX-C1 & 20 & - & $5.1(3.9-6.7)$ & $19.4(18-20.9)$ & $17.8(16.3-19.5)$ \\
GTX-C2 & 20 & - & $5.1(3.9-6.5)$ & $20(18.5-21.6)$ & $18.4(16.8-20.1)$ \\
GTX1 & 20 & $10.8(0.7)$ & $4.4(3.1-6.3)$ & $3.6(3.3-4.0)$ & $3.3(3.0-3.7)$ \\
GTX2 & 20 & $13.6(1)$ & $4.5(3.3-6.2)$ & $3.5(3.2-3.9)$ & $3.2(2.9-3.6)$ \\
\hline
\end{tabular}

probability that GTX-C1 and GTX-C2 will last longer than GTX1 and GTX2 was $100 \%$ (all were statistically significant).

However, when control groups were compared between them, the probability that unused but sterilized GT will last longer than unused GT was also statistically significant (66\%), although it was not for GTX (54\%). And the same happened when experimental groups were compared; the probability that GT1 will last longer that GT2 was $62 \%$ (statistically significant), and the probability that GTX1 will last longer than GTX2 was 52\% (not statistically significant).

When mean life was compared between brands, it was significantly higher in unused files for GTX than for GT, but it was significantly higher for GT than for
GTX in used files. GTX-C1 and GTX-C2 will last longer than GT-C1 GT-C2 with a probability of $75 \%$ and $65 \%$, respectively. However, the probability that GT will last longer than GTX was $72 \%$ after shaping three molars (GT1 compared with GTX1) and 68\% after shaping four molars (GT2 compared with GTX2).

\section{Discussion}

The cyclic fatigue device and the statistical approach used in this study have been discussed previously (Arias et al. 2012). Cyclic fatigue resistance of GT and GTX files was tested using new files and after being used in real clinical conditions.

This study tested static cyclic fatigue in a hardened steel canal. The design of the canals did not 
reproduce clinical conditions. Many studies tested cyclic fatigue in metal devices: from the Cheung's design in which the file is constricted into a curvature delimited by three stainless steel pins (Cheung \& Darvell 2007) to different types of simulated canals (Grande et al. 2006, Gambarini et al. 2012). The benefit of the device used in this study is that the fulcrum point for the fatigue test can be decided by the operator.

The application of cyclic fatigue at $3 \mathrm{~mm}$ from the tip was decided because GT and GTX files are supposed to work only apically when the shaping protocol used in patients is followed. This protocol mixed two different NiTi rotary systems: ProTaper and GT/GTX to gain efficacy when preparing coronal and middle thirds of canals, whilst being conservative in the apical third.

The use of the whole sequence of ProTaper has been reported to have several drawbacks. Canals shaped with rotary ProTaper were progressively straightened after the use of F1 producing transportation (Zhang et al. 2008). Hybrid instrumentation has been claimed to be a valid alternative to achieve larger apical diameters diminishing the risk of procedural errors (Setzer et al. 2010). It has been recommended that the combination of S1 and S2 with other less tapered and more flexible files avoids apical transportation (Javaheri \& Javaheri 2007).

Finishing canal shaping with GT or GTX has advantages. ProTaper and GT have been shown to be equally effective in root canal debridement (Williamson et al. 2009), but dentinal damage was reduced from $16 \%$ when the shaping procedure was completely performed with ProTaper to $4 \%$ when it was with GT (Bier et al. 2009) and GTX exhibited superior performance than ProTaper regarding both canal transportation and centring (Hashem et al. 2012). GTX NiTi rotary files and Profile GT files performed similarly in an extracted tooth model (Iqbal et al. 2010).

In this study, files were used in up to four molars following recommendations of Yared et al. (2000), who analysed cyclic fatigue in Profile rotary files (with an U-shaped cross-section) and concluded that they could be used safely in up to four human molars.

The results of this laboratory study revealed that unused GTX files have a longer mean life than unused GT. To date, most of the published studies that compare cyclic fatigue of unused GT and GTX files suggest that rotary files made from the newly developed M-wire alloy have better flexural cyclic fatigue resistance than files of similar design and size made from the conventional nickel-titanium alloy (Larsen et al. 2009, da Cunha Peixoto et al. 2010, Solaiman et al. 2010). Only two studies did not find that new M-Wire instruments had better cyclic fatigue resistance when compared with Profile, GT files (Kramkowski \& Bahcall 2009) or K3 (Gambarini et al. 2008). Moreover, several studies have shown that not only GTX, but also other files made of R-phase and M-Wire alloys are more resistant to cyclic fatigue than files made of traditional NiTi (Gambarini et al. 2011, Bouska et al. 2012).

In particular, a recent study reported that unused GTX files are more flexible and capable of stress relief at the most critical sections than unused GT files, suggesting that they would have a lower risk of fracture inside the root canals during clinical use (Montalvao \& Alçada 2011).

It is not fully understood why a theoretically more resistant new alloy may lose its superiority when it ages, but the results of this study revealed lower resistance to CF of GTX than of GT files after being used in clinical conditions. da Cunha Peixoto et al. (2010) observed that pitch lengths were larger in GTX instruments when compared with similar GT instruments, whereas the diameter of GTX files tended to be smaller. It is difficult to anticipate whether this intrinsic characteristic influenced the results of this study, but it may be that wear on the blades that the shaping procedure produces could diminish the advantages of the design of GTX and of the superior alloy and therefore have an influence in the results. At the same time, the results of this study also showed that although GT used files had better cyclic fatigue resistance than GTX files, GT files age more rapidly than GTX (resistance of GT files was significantly affected by the number of canals previously shaped, but this difference was not statistically significant in GTX files). It seems that although resistance to cyclic fatigue is significantly higher in GT than in GTX files, the cyclic fatigue resistance that is expected in GT files when it is used to shape a fourth molar is significantly lower than when it was used in the third. On the other hand, the expected cyclic fatigue resistance in GTX did not significantly differ when shaping the root canals of a third or a fourth molar.

Comparison of cyclic fatigue resistance between new and used instruments has been studied with different NiTi rotary files and various studies agree in the higher resistance of new instruments (Gambarini 
2001, Bahia \& Buono 2005). It seems accepted that prolonged reuse of NiTi rotary instruments substantially affects their fatigue (Fife et al. 2004, Aydin et al. 2010). Once again, the results of the present study support that finding.

Debate continues over whether autoclave sterilization or clinical usage is the leading cause of the ageing of NiTi alloys. In the present study, two different methods were used to age files: autoclave sterilization alone and shaping of canals in vivo and autoclave sterilization together. Cyclic fatigue resistance did not decrease in files that were only sterilized, but it decreased drastically when they were used in vivo. Irrigants used during shaping or the procedure itself seemed to diminished cyclic fatigue resistance. In this study, $\mathrm{NaOCl}$ was used with a higher concentration $(5.25 \%)$, but given that the same irrigant protocol and sterilization process were used with both GT and GTX, it is probable that a different rates of wear of the blades could be responsible for the lower resistance to cyclic fatigue of used GTX files when compared with used GT files. This finding and the fact that the use of $2.5 \% \mathrm{NaOCl}$ did not alter cyclic fatigue of Profile files (Yared et al. 1999, 2000), supports the hypothesis that shaping procedures are the main factor in the decline of cyclic fatigue resistance of files.

Previous studies have also found that autoclave sterilization did not affect cyclic fatigue behaviour of new GT series X files (Hilfer et al. 2011) and enhanced cyclic fatigue resistance of new K3XF files (Plotino et al. 2012), although it reduced GT series $X$ torsional strength significantly (King et al. 2012). In the present study, cyclic fatigue resistance of GTX was not significantly affected by autoclave sterilization, but, unexpectedly, cyclic fatigue resistance of GT was longer when new files were sterilized. This difference suggests that autoclave sterilization does not enhance the alloy when it has been previously altered with heat (M-Wire), but probably the heat of the sterilization process affects conventional NiTi.

\section{Conclusions}

GT series X files were associated with higher cyclic fatigue resistance than GT when they were unused or unused and sterilized, but GT were significantly more resistant to cyclic fatigue after clinical usage than GT series $\mathrm{X}$ files at $3 \mathrm{~mm}$ from the tip. Nevertheless, and in accordance with the claim of the designer of the file, the improvements in GT series $\mathrm{X}$ files do not allow clinicians to shape more canals than they used to shape with GT files (Buchanan 2008).

\section{Acknowledgements}

The authors thank Dentsply Maillefer for providing the instruments for the control groups.

\section{References}

Arias A, Perez-Higueras JJ, de la Macorra JC (2012) Differences in cyclic fatigue resistance at apical and coronal levels of Reciproc and WaveOne new files. Journal of Endodontics 38, 1244-8.

Aydin C, Inan U, Tunca YM (2010) Comparison of cyclic fatigue resistance of used and new Race instruments. Oral Surgery, Oral Medicine, Oral Pathology, Oral Radiology \& Endodontics 109, e131-4.

Bahia MGA, Buono VTL (2005) Decrease in the fatigue resistance of nickel-titanium rotary instruments after clinical use in curved root canals. Oral Surgery, Oral Medicine, Oral Pathology, Oral Radiology, \& Endodontics 100, 249-55.

Bier CA, Shemesh H, Tanomaru-Filho M, Wesselink PR, Wu MK (2009) The ability of different nickel-titanium rotary instruments to induce dentinal damage during canal preparation. Journal of Endodontics 35, 236-8.

Bird DC, Chambers D, Peters OA (2009) Usage parameters of nickel-titanium rotary instruments: a survey of endodontists in the united states. Journal of Endodontics 35, 1193-7.

Bouska J, Justman B, Williamson A, DeLong C, Qian F (2012) Resistance to cyclic fatigue failure of a new endodontic rotary file. Journal of Endodontics 38, 667-9.

Buchanan LS (2008) The new gt series x rotary shaping system: objectives and technique principles. Dentistry Today 27, 70-4.

Cheung GSP, Darvell BW (2007) Fatigue testing of a NiTi rotary instrument. Part 1: strain-life relationship. International Endodontic Journal 40, 612-18.

da Cunha Peixoto IF, Pereira ES, da Silva JG, Viana AC, Buono VT, Bahia MG (2010) Flexural fatigue and torsional resistance of Profile GT and Profile GT series X instruments. Journal of Endodontics 36, 741-4.

Fife D, Gambarini G, Britto LR (2004) Cyclic fatigue testing of protaper NiTI rotary instruments after clinical use. Oral Surgery, Oral Medicine, Oral Pathology, Oral Radiology \& Endodontics 97, 251-6.

Gambarini G (2001) Cyclic fatigue of nickel-titanium rotary instruments after clinical use with low-and high-torque endodontic motors. Journal of Endodontics 27, 772-4.

Gambarini G, Grande NM, Plotino G, et al. (2008) Fatigue resistance of engine-driven rotary nickel-titanium instruments produced by new manufacturing methods. Journal of Endodontics 34, 1003-5. 
Gambarini G, Plotino G, Grande NM, Al-Sudani D, De Luca M, Testarelli L (2011) Mechanical properties of nickeltitanium rotary instruments produced with a new manufacturing technique. International Endodontic Journal 44, 337-41.

Gambarini G, Gergi R, Naaman A, Osta N, Al Sudani D (2012) Cyclic fatigue analysis of twisted file rotary NiTi instruments used in reciprocating motion. International Endodontic Journal 45, 802-6.

Glosson CR, Haller RH, Dove SB, Delrio CE (1995) A comparison of root-canal preparations using NiTi hand, NiTi engine-driven, and K-Flex endodontic instruments. Journal of Endodontics 21, 146-51.

Grande NM, Plotino G, Pecci R, Bedini R, Malagnino VA, Somma F (2006) Cyclic fatigue resistance and three-dimensional analysis of instruments from two nickel-titanium rotary systems. International Endodontic Journal 39, 755-63.

Hashem AA, Ghoneim AG, Lutfy RA, Foda MY, Omar GA (2012) Geometric analysis of root canals prepared by four rotary NiTi shaping systems. Journal of Endodontics 38, 996-1000.

Hilfer PB, Bergeron BE, Mayerchak MJ, Roberts HW, Jeansonne BG (2011) Multiple autoclave cycle effects on cyclic fatigue of nickel-titanium rotary files produced by new manufacturing methods. Journal of Endodontics 37, 72-4.

Iqbal MK, Floratos S, Hsu YK, Karabucak B (2010) An in vitro comparison of Profile GT and GTX nickel-titanium rotary instruments in apical transportation and length control in mandibular molar. Journal of Endodontics 36, 302-4.

Javaheri HH, Javaheri GH (2007) A comparison of three ni-ti rotary instruments in apical transportation. Journal of Endodontics 33, 284-6.

Johnson E, Lloyd A, Kuttler S, Namerow K (2008) Comparison between a novel nickel-titanium alloy and 508 nitinol on the cyclic fatigue life of Profile 25/.04 rotary instruments. Journal of Endodontics 34, 1406-9.

Kell T, Azarpazhooh A, Peters OA, El-Mowafy O, Tompson B, Basrani B (2009) Torsional profiles of new and used 20/.06 GT series x and GT rotary endodontic instruments. Journal of Endodontics 35, 1278-81.

King JB, Roberts HW, Bergeron BE, Mayerchak MJ (2012) The effect of autoclaving on torsional moment of two nickel-titanium endodontic files. International Endodontic Journal 45, 156-61.

Kramkowski TR, Bahcall J (2009) An in vitro comparison of torsional stress and cyclic fatigue resistance of Profile GT and Profile GT series X rotary nickel-titanium files. Journal of Endodontics 35, 404-7.

Larsen CM, Watanabe I, Glickman GN, He J (2009) Cyclic fatigue analysis of a new generation of nickel titanium rotary instruments. Journal of Endodontics 35, 401-3.

Montalvao D, Alçada FS (2011) Numeric comparison of the static mechanical behaviour between of Profile GT and
Profile GT series X rotary nickel-titanium files. Journal of Endodontics 37, 1158-61.

Ounsi HF, Salameh Z, Al-Shalan T, et al. (2007) Effect of clinical use on the cyclic fatigue resistance of protaper nickel-titanium rotary instruments. Journal of Endodontics 33, 737-41.

Ounsi HF, Franciosi G, Paragliola R, et al. (2011) Comparison of two techniques for assessing the shaping efficacy of repeatedly used nickel-titanium rotary instruments. Journal of Endodontics 37, 847-50.

Plotino G, Grande NM, Sorci E, Malagnino VA, Somma F (2006) A comparison of cyclic fatigue between used and new mtwo Ni-Ti rotary instruments. International Endodontic Journal 39, 716-23.

Plotino G, Costanzo A, Grande NM, Petrovic R, Testarelli L, Gambarini G (2012) Experimental evaluation on the influence of autoclave sterilization on the cyclic fatigue of new nickel-titanium rotary instruments. Journal of Endodontics 38, 222-5.

Sattapan B, Nervo GJ, Palamara JE, Messer HH (2000) Defects in rotary nickel-titanium files after clinical use. Journal of Endodontics 26, 161-5.

Setzer FC, Kwon TK, Karabucak B (2010) Comparison of apical transportation between two rotary file systems and two hybrid rotary instrumentation sequences. Journal of Endodontics 36, 1226-9.

Short JA, Morgan LA, Baumgartner JC (1997) A comparison of canal centering ability of four instrumentation techniques. Journal of Endodontics 23, 503-7.

Solaiman MSA-H, Fahad AA, Riyadh IA (2010) Evaluation of cyclic flexural fatigue of M-wire nickel-titanium rotary instruments. Journal of Endodontics 36, 305-7.

Walia H, Brantley WA, Gerstein H (1988) An initial investigation of the bending and torsional properties of nitinol root canal files. Journal of Endodontics 14, 346-51.

Williamson AE, Sandor AJ, Justman BC (2009) A comparison of three nickel titanium rotary systems, Endosequence, Protaper universal, and Profile GT, for canal-cleaning ability. Journal of Endodontics 35, 107-9.

Yared GM, Dagher FEB, Machtou P (1999) Cyclic fatigue of profile rotary instruments after simulated clinical use. International Endodontic Journal 32, 115-19.

Yared GM, Dagher FEB, Machtou P (2000) Cyclic fatigue of profile rotary instruments after clinical use. International Endodontic Journal 33, 204-7.

Ye J, Gao Y (2012) Metallurgical characterization of M-Wire nickel-titanium shape memory alloy used for endodontic rotary instruments during low-cycle fatigue. Journal of Endodontics 38, 105-7.

Zhang L, Luo HX, Zhou XD, Tan H, Huang DM (2008) The shaping effect of the combination of two rotary nickeltitanium instruments in simulated s-shaped canals. Journal of Endodontics 34, 456-8. 